\title{
Treatment of Osteosarcoma Patients in the Philippine General Hospital during the COVID-19 Outbreak
}

\author{
Czar Louie Gaston, MD, ${ }^{1}$ Hazel Valerie Yu, MD, ${ }^{2}$ Emileo Dacanay, MD, ${ }^{1}$ Cesar Cipriano Dimayuga, MD, ${ }^{1}$ \\ Jochrys Estanislao, MD, ${ }^{2}$ Pamela Fajardo, MD, ${ }^{2}$ Albert Jerome Quintos, MD, ${ }^{1}$ \\ Donnel Alexis Rubio, MD, ${ }^{1}$ Edward Wang, MD ${ }^{1}$ and Ana Patricia Alcasabas, MD² \\ ${ }^{1}$ Department of Orthopedics, Philippine General Hospital, University of the Philippines Manila \\ ${ }^{2}$ Department of Pediatrics, Philippine General Hospital, University of the Philippines Manila
}

\begin{abstract}
Objectives. The ongoing Coronavirus disease 2019 (COVID-19) pandemic has disrupted healthcare systems worldwide. This study aimed to document the effect of COVID-19 on osteosarcoma treatment pathways in the Philippine General Hospital (PGH) and determine if there were any delays.
\end{abstract}

Method. A retrospective review of osteosarcoma patients treated at the PGH from January 1, 2019 - January 1, 2020 (pre-COVID-19) was compared to those treated during the COVID-19 pandemic from March 1, 2020 September 1, 2020. Rates of diagnosed osteosarcoma, admission for chemotherapy, admission for surgery, treatment abandonment, metastatic disease on presentation, 1-year mortality, and amputation were calculated and compared between the two groups.

Results. From March to September 2020, 11 newly diagnosed osteosarcoma patients sought consult at the PGH. Only one patient sought consult during the initial 3-4 months of the study, suggesting that patients delayed seeking healthcare during the period of enhanced community quarantine. Patients seen during the pandemic had a higher rate of metastatic disease on presentation, reflecting the delay in diagnosis. Due to COVID-19 restrictions early in the pandemic, osteosarcoma patients were coordinated and referred to outside hospitals for intravenous chemotherapy and surgery. Normalization of services (hospital admissions, limb salvage surgeries) were seen at the later stages of the study, corresponding to the loosening of the quarantine.

Conclusions. Osteosarcoma patients experienced delays in seeking consult, diagnosis, and treatment at the PGH due to the COVID-19 pandemic. Early indicators suggest worse outcomes for these patients due to the delays. Strategies employed during the pandemic, such as networking of care and telemedicine, may help in future outbreaks.

Key Words: osteosarcoma, coronavirus, COVID-19, pandemic

\section{INTRODUCTION}

Conventional osteosarcoma is a high-grade malignancy that commonly affects children, adolescents, and young adults with reported 5-year overall survival rates of 50-70\%. Multidisciplinary management is mandatory with multiagent chemotherapy and surgery given over 6-8 months. Timely treatment and coordination of different medical subspecialties are essential to avoid treatment abandonment

Corresponding author: Czar Louie Gaston, MD

Department of Orthopedics

Philippine General Hospital

University of the Philippines Manila

Taft Avenue, Ermita, Manila 1000, Philippines

Email: clgaston@up.edu.ph and improve cancer outcomes. ${ }^{1}$

Since December 2019, Coronavirus Disease-2019 (COVID-19), a viral infectious disease, has gradually spread across multiple countries, causing low-to-moderate mortality and infecting millions worldwide. ${ }^{2}$ Due to the 
risk of host-to-host transmission, strategies to contain the spread of the disease have included social distancing and strict home quarantine to avoid getting into contact with the virus. ${ }^{3}$ Despite these social measures, frontline health institutions such as hospitals and tertiary referral centers have been overwhelmed with suspected COVID-19 patients. In trying to address the burden of the virus, health systems in developing countries with dense communities may have to do so at the expense of other healthcare services. ${ }^{4}$

In the Philippines, hospitals have adopted rigorous screening of patients and concentration of their services in handling COVID-19 cases. The Philippine College of Surgeons gave recommendations for canceling elective nonurgent surgeries and minimizing outpatient clinics in March 2020 to address the COVID-19 pandemic. ${ }^{5}$ These measures aimed to decrease patients, family members, and healthcare workers' exposure to COVID-19 and conserve health system resources for COVID-19 patients. How these strategies could affect health-seeking behavior and treatment of nonCOVID-19 patients with other conditions such as cancer is currently unknown.

There is limited data for the treatment of cancer during the COVID-19 outbreak. In a cohort of 1,590 patients, Liang et al. found that $1 \%$ had a cancer diagnosis and were under active treatment or follow-up. ${ }^{6}$ These patients were at a higher risk for ICU admission or death due to COVID-19. The authors recommended intentional postponement of chemotherapy or surgery for stable cancer in endemic areas. Liu et al. reported that hospital visits may be delayed, converted to online follow-up, or both after careful discussion of the risks between doctors and breast cancer patients. ${ }^{7}$

Delays in chemotherapy and surgery in osteosarcoma in low-to-middle income countries have been shown to lead to poorer outcomes and should be avoided. ${ }^{1,8}$ However, the current COVID-19 crisis has put unforeseen pressure on hospitals worldwide, leading to delays in treatment for osteosarcoma patients.

This cross-sectional study aims to document the effect of COVID-19 on osteosarcoma treatment pathways in a tertiary referral center in the Philippines. We hypothesize that the COVID-19 outbreak affected how Philippine healthcare systems treat other non-COVID-19 conditions and negatively affect osteosarcoma patients. By identifying deficiencies in diagnosis, delivery of chemotherapy, and surgery, we hope to offer recommendations and strategies to improve osteosarcoma care in the Philippine General Hospital (PGH) and the Philippines in future outbreaks.

\section{METHODOLOGY}

Chart review of high-grade osteosarcoma patients treated at the PGH from January 1, 2019, and September 1, 2020, including the COVID-19 pandemics period from March 1, 2020, onwards, was performed. Inclusion criteria were all high-grade osteosarcoma patients of the extremity and pelvis confirmed by histopathology review by the Department of Pathology of the PGH and 1) they are already diagnosed and undergoing active treatment (chemotherapy and surgery) before March 1, 2020 (pre-COVID-19 group) and 2) they were diagnosed and treated at the PGH from March 1, 2020, and September 1, 2020 (COVID-19 group). Patients without biopsy confirmation of high-grade osteosarcoma, low-grade osteosarcomas, and cases referred only for opinion were excluded from the study.

Osteosarcoma patients already diagnosed and undergoing active treatment before March 1, 2020, were monitored for any admission delays for chemotherapy and surgery. Reasons for delays were classified as COVID-19 or nonCOVID-19 related. Directly COVID-19-related reasons include lack of hospital beds, lack of operating room facility, transportation issues to $\mathrm{PGH}$ due to lockdown/quarantine, and recent COVID-19 infection.

Treatment abandonment was documented as per prior protocol, and desertion reasons were investigated and classified as COVID-19 or non-COVID-19 related. ${ }^{9}$ Osteosarcoma patients referred and transferred to other institutions for chemotherapy and surgery by the Musculoskeletal Oncology team of the PGH due to COVID-19 reasons were also recorded.

Virtual/online communication for routine outpatient health care of patients is currently performed using Skype or Zoom in keeping with social distancing directives to limit contamination by COVID-19. ${ }^{10}$ Consent for the study was taken by members of the healthcare and research team [CLG, HY, ED] during the online communication for patient follow-up and confirmed with properly signed informed consent forms returned by email. No study-related interviews other than informed consent was conducted.

Metastases on presentation, 1-year mortality rate, and amputation rate of osteosarcoma patients diagnosed and treated between March 1, 2020 - September 1, 2020 (COVID-19 group) were documented as markers for treatment delays.

Rates of diagnosed osteosarcoma, admission for chemotherapy, admission for surgery, treatment abandonment, follow-up, 1-year mortality, and amputation were calculated for the osteosarcoma COVID-19 group and compared to the pre-COVID-19 group by chi-square and students t-test with a $\mathrm{p}<0.05$ considered significant.

This study was conducted following the Helsinki Declaration. This study was approved by the University of the Philippines Manila REB and received grant funding from the UPM-PGH Expanded Hospital Research Office.

\section{RESULTS}

From March 1 to September 1, 2020, 11 newly diagnosed osteosarcoma patients sought consult at the PGH (Table 1). Demographic data of osteosarcoma patients diagnosed during the COVID-19 pandemic was similar to 
Treatment of osteosarcoma patients during COVID-19 Outbreak

Table 1. Osteosarcoma patients in the PGH diagnosed during COVID-19 outbreak compared to Pre-COVID-19 group

\begin{tabular}{|c|c|c|c|}
\hline & $\begin{array}{l}\text { COVID-19 } \\
\text { outbreak }\end{array}$ & $\begin{array}{r}\text { Pre-COV } \\
\text { (January 1, } 2019 \text { - }\end{array}$ & 1,2020) \\
\hline Diagnosed osteosarcomas (n) & 11 & 17 & \\
\hline Age (years) & 17.6 (SD 7.3) & 13.9 (SD 3.4) & $p=0.08$ \\
\hline $\begin{array}{l}\text { Residence (n) } \\
\text { NCR } \\
\text { Province } \\
\end{array}$ & $\begin{array}{l}7 / 11 \\
4 / 11 \\
\end{array}$ & $\begin{array}{c}10 / 17 \\
7 / 17 \\
\end{array}$ & $p=0.25$ \\
\hline $\begin{array}{l}\text { Tumor Site }(\mathrm{n}) \\
\text { Extremity } \\
\text { Pelvis } \\
\end{array}$ & $\begin{array}{c}10 / 11 \\
1 / 11 \\
\end{array}$ & $\begin{array}{c}17 / 17 \\
0 / 17 \\
\end{array}$ & $p=0.96$ \\
\hline Chemotherapy admissions ( $\mathrm{n}$ ) & 30 & 80 & $p=0.02$ \\
\hline Surgery admissions (n) & 10 & 14 & $p=0.19$ \\
\hline Treatment abandonment (n) & 2 & 0 & $p=0.13$ \\
\hline Metastatic disease on presentation & $73 \%(n=8 / 11)$ & $41 \%(n=7 / 17)$ & $p=0.04$ \\
\hline 1-year mortality rate & $36 \%(n=4 / 11)$ & $41 \%(n=7 / 17)$ & $p=0.91$ \\
\hline Amputation rate & $80 \%(n=8 / 10)$ & $78 \%(n=11 / 14)$ & $p=0.76$ \\
\hline Outside hospital referral rate & $55 \%(n=6 / 11)$ & $5 \%(n=1 / 17)$ & $p=0.22$ \\
\hline
\end{tabular}

NCR: National Capital Region, $p<0.05$ considered significant

Table 2. Delays in treatment of osteosarcoma patients in the PGH during COVID-19 outbreak

\begin{tabular}{lcl} 
Chemotherapy delay & Number $(n)$ & \multicolumn{1}{c}{ Reasons } \\
COVID-19 related & $11 / 14$ & $\begin{array}{l}\text { COVID-19 center directive }(n=5) \\
\text { Lack of transportation }(n=3) \\
\text { COVID-19 infection }(n=1)\end{array}$ \\
Fon-COVID-19 related delay & 9 & Financial $(n=2)$ \\
\hline $\begin{array}{c}\text { Surgery delays } \\
\text { COVID-19 related }\end{array}$ & 2 & $\begin{array}{l}\text { COVID center directive }(n=6) \\
\text { Lack of transportation }(n=1)\end{array}$ \\
Non-COVID-19 related delay & $7 / 14$ & Financial $(n=1)$ \\
\hline
\end{tabular}

pre-COVID-19 in terms of the locality of residence and tumor site. A trend in increasing age at diagnosis was seen for patients seeking consult during the outbreak. Compared to 2019 , the average number of admissions for chemotherapy per patient during the COVID-19 pandemic significantly decreased (2.75 vs. 4.8, $\mathrm{p}=0.02)$. During the enhanced community quarantine in Metro Manila (March to June 2020), no patient was admitted for in-patient intravenous chemotherapy due to COVID-19 restrictions. Two patients with metastatic disease were treated with oral etoposide administered at home. In contrast, one newly diagnosed non-metastatic patient was referred to another institution to receive standard neoadjuvant doxorubicin and cisplatin.

Although mortality rate and amputation rate were similar in the two cohorts (Table 2), the proportion of osteosarcoma patients presenting with metastatic disease at diagnosis was significantly higher during the COVID-19 pandemic.

Osteosarcoma patients experienced delays in receiving chemotherapy and surgery during the COVID-19 pandemic (Table 2). The designation of PGH as a COVID-19 center directly affected the hospital's ability to give chemotherapy and surgery to osteosarcoma patients, especially during the
ECQ period. Lack of transportation due to the quarantine affected three patients seeking treatment in PGH regardless of their geographical location (Manila, Valenzuela, Bicol). One patient's admission for chemotherapy was delayed due to a positive COVID-19 infection.

From March to May 2020, six osteosarcoma patients for surgery were referred to outside institutions due to restrictions on performing elective surgery at PGH being a COVID-19 center. The majority of surgeries completed during the early stages of the pandemic were amputations. The only two limb salvage surgeries done in the COVID-19 group were on patients diagnosed in August to September 2020, both of which had no delays and restrictions in neoadjuvant chemotherapy admissions.

\section{DISCUSSION}

Since being classified as a pandemic by the WHO in March 2020, COVID-19 has drastically affected everyday life for citizens worldwide, with deleterious effects on those directly and indirectly affected by the virus. Due to governmental strategies to limit transmission of the virus, 
healthcare and health-seeking behavior of those with other diseases, including cancer, have been adversely influenced.

Cancellation of cancer services during this COVID-19 outbreak was reported worldwide. A survey of orthopedic oncologists revealed that $20 \%$ had stopped treating lifethreatening sarcomas during the COVID-19 pandemic. ${ }^{11}$ Early reports cited adverse outcomes of cancer patients if they contracted COVID-19., This, along with the stoppage of elective hospital services to redirect resources to treat COVID-19 infections and limit transmission of the virus, led to the reorganization of cancer care in many hospital settings, including the PGH. ${ }^{12}$

The present study showed that in a tertiary referral hospital designated as a COVID-19 center, osteosarcoma patients experienced delays in receiving chemotherapy and surgery. In response to the hospital restrictions in providing services such as admission for intravenous chemotherapy and performing elective surgery, the Musculoskeletal Tumor Unit of the Department of Orthopedics and HemaOncology Unit of the Department of Pediatrics of the PGH had to coordinate the referral of existing osteosarcoma patients to outside institutions for their treatment during the height of the pandemic. From March to May 2020, there were no direct hospital admissions for chemotherapy and surgery for osteosarcoma in PGH. Only one new osteosarcoma patient was seen at the hospital emergency room and promptly referred for workup and treatment to another hospital during this period.

Despite only having one new osteosarcoma patient seeking consult in 3 months, by the end of the 6-month study period, the total number of osteosarcoma patients seen was 11 , which is comparable with the average census of the PGH Musculoskeletal Tumor Unit of 1-2 new osteosarcomas/ month (Table 1). This data suggests that patients delayed seeking consult for their condition. Reasons for the delays include the difficulty in traveling around Metro Manila due to the enhanced community quarantine and PGH being designated as a COVID-19 center.

The delays in seeking treatment were also manifested by the significantly increased metastatic disease rate on the presentation by the osteosarcoma patients diagnosed during the pandemic. Over $70 \%$ already had demonstrable lung metastases upon diagnosis. Although 1-year mortality rates were similar to the pre-COVID-19 cohort at the time of analysis, this was likely due to the short follow-up period and their advanced stage on diagnosis portends a dismal prognosis for these patients experienced delays in treatment due to the pandemic. The high amputation rate at the start of the COVID-19 outbreak seen in our study was also described in other institutions worldwide, although it became apparent with later studies that limb salvage surgeries were appropriate to perform during the pandemic. ${ }^{11,13}$

Due to the difficulty of PGH to provide direct services to osteosarcoma patients during the first 2-3 months of the pandemic, rapid coordination through networking and referral of care to other specialist hospitals inside Metro Manila and in the provinces was crucial. ${ }^{14}$ Avenues for online communication with patients using telemedicine were also established, which provided easy access to a healthcare specialist. These strategies were essential to avoid further delays in treatment. They may be applied to osteosarcoma patients' multidisciplinary treatment in future pandemics and instances where hospital services are again similarly affected.

This study was limited by the possibility that the numbers of osteosarcoma patients who consulted or were referred by outside institutions may have been affected by PGH being designated as a COVID-19 center. However, these restrictions made the results of this study more critical as we have now been able to gauge the early effects of a pandemic on non-COVID-19 health care in a tertiary government center. The limited follow-up (less than one year) of the COVID-19 group is another limitation of the study. It may be addressed with another study looking into the continuing long-term effects of the ongoing COVID-19 pandemic.

\section{CONCLUSIONS}

Osteosarcoma patients experienced delays in seeking consult, diagnosis, and treatment at the PGH due to the COVID-19 pandemic. Delays primarily occurred during the period of enhanced community quarantine, and there was evidence that services normalized during the later stages of the study. Early indicators suggest worse survival outcomes for patients who experienced delays due to the pandemic. Future studies are recommended to monitor the long-term effects on osteosarcoma care, if any. Strategies employed during the pandemic, such as networking of care and telemedicine, may help in future outbreaks.

\section{Statement of Authorship}

All authors participated in the data collection and analysis and approved the final version submitted.

\section{Author Disclosure}

All authors declared no conflicts of interest.

\section{Funding Source}

This paper was funded by the 2020 Paramedical and Faculty Research Grant.

\section{REFERENCES}

1. Vasquez L, Silva J, Chavez S, Zapata A, Diaz R, Tarrillo F, et al. Prognostic impact of diagnostic and treatment delays in children with osteosarcoma. Pediatr Blood Cancer. 2020 Apr;67(4):e28180. doi: 10.1002/pbc.28180. Epub 2020 Jan 11.

2. Wu YC, Chen CS, Chan YJ. The outbreak of COVID-19: An overview. J Chin Med Assoc.2020;83(3):217-20. doi: 10.1097/JCMA. 0000000000000270 . PMID: 32134861; PMCID: PMC7153464.

3. Adhikari SP, Meng S, Wu YJ, Mao YP, Ye RX, Wang QZ, et al. Epidemiology, causes, clinical manifestation and diagnosis, prevention 
and control of coronavirus disease (COVID-19) during the early outbreak period: a scoping review. Infect Dis Poverty. 2020 Mar 17;9(1):29. doi: 10.1186/s40249-020-00646-x. PMID: 32183901; PMCID: PMC7079521.

4. Velavan TP, Meyer CG. The COVID-19 epidemic. Trop Med Int Health. 2020 Mar;25(3):278- 80. doi: 10.1111/tmi.13383. Epub 2020 Feb 16. PMID: 32052514; PMCID: PMC7169770.

5. Salud JAM. Philippine College of Surgeons Recommendations for Elective Surgery. March 14, 2020. Date accessed April 2020.

6. Liang W, Guan W, Chen R, Wang W, Li J, Xu K, et al. Cancer patients SARS-CoV-2 infection: a nationwide analysis in China. Lancet Oncol 2020;21:335-7. doi: 10.1016/S1470-2045(20)30096-6. Epub $2020 \mathrm{Feb} 14$

7. Liu BL, Ma F, Wang JN, Fan Y, Mo HN, Xu BH. [Health management of breast cancer patients outside the hospital during the outbreak of 2019 novel coronavirus disease]. Zhonghua Zhong Liu Za Zhi. 2020 Feb 26;42(0):E002.

8. Abou Ali B, Salman M, Ghanem KM, Boulos F, Haidar R, Saghieh S, Akel S, et al. Clinical Prognostic Factors and Outcome in Pediatric Osteosarcoma: Effect of Delay in Local Control and Degree of Necrosis in a Multidisciplinary Setting in Lebanon. J Glob Oncol. 2019 Apr;5:1-8. doi: 10.1200/JGO.17.00241. PMID: 30946633; PMCID: PMC6528739.

9. Mostert S, Arora RS, Arreola M, Bagai P, Friedrich P, Gupta S, et al. Abandonment of treatment for childhood cancer: position statement of a SIOP PODC Working Group. Lancet Oncol. 2011; 12(8): 719-20. doi: 10.1016/S1470-2045(11)70128-0. Epub 2011 Jun 28. PMID: 21719348.
10. Greenhalgh T, Shaw S, Wherton J, Vijayaraghavan S, Morris $\mathrm{J}$, Bhattacharya S, et al. Real-World Implementation of Video Outpatient Consultations at Macro, Meso, and Micro Levels: MixedMethod Study. J Med Internet Res. 2018 Apr 17;20(4):e150. doi: 10.2196/jmir.9897. PMID: 29625956; PMCID: PMC5930173.

11. Thaler M, Khosravi I, Leithner A, Papagelopoulos P, Ruggieri P. Impact of the COVID-19 pandemic on patients suffering from musculoskeletal tumours. Int Orthop 2020 Aug;44(8):1503-9. doi: 10.1007/s00264-020-04636-4. Epub 2020 May 26.

12. Mendoza MJL, Tan HNC, Hernandez ARB, Dala BCA, Sacdalan DBL, Sacdalan DL, et al. Medical oncology care amidst the COVID-19 pandemic at the National University Hospital in the Philippines. Ecancermedicalscience. 2020 Jun 30;14:1066. doi: 10.3332/ecancer. 2020.1066. PMID: 32728382; PMCID: PMC7373646..

13. Stevenson JD, Evans S, Morris G, Tillman R, Abudu A, Jeys L, Parry M. Mortality of high-risk orthopaedic oncology patients during the COVID-19 pandemic: A prospective cohort study. J Surg Oncol. 2020 Jul 15:10.1002/jso.26127. doi: 10.1002/jso.26127. Epub ahead of print. PMID: 32668015; PMCID: PMC7405118.

14. Gaston CLL, Pag-Ong JP, Dacanay E, Quintos AJ. Radical change in osteosarcoma surgical plan due to COVID-19 pandemic. BMJ Case Rep. 2020 Jul 8;13(7):e237197. doi: 10.1136/bcr-2020-237197. PMID: 32641312; PMCID: PMC7348652.

\title{
Have you read the current trends in
} Medical and Health Research in the Philippines?

\section{Acta Medica Philippina The National Health Science Journal}

\author{
Access Online: www.actamedicaphilippina.upm.edu.ph
}

